\section{A New Criterion of Delay-Dependent Asymptotic Stability for Hopfield Neural Networks With Time Delay}

Shaoshuai Mou, Huijun Gao, James Lam, and Wenyi Qiang (semidefinite). $I$ and 0 denote the identity matrix and zero matrix with compatible dimensions, and $\operatorname{diag}\{\ldots\}$ stands for a block-diagonal matrix. Matrices are assumed to be compatible for algebraic operations if their dimensions are not explicitly stated.

\section{PROBLEM Formulation}

Consider the following continuous HNN with time delay:

$$
\dot{x}_{i}(t)=-a_{i} x_{i}(t)+\sum_{j=1}^{n} b_{i j} f_{j}\left(x_{j}(t-\tau)\right)+c_{i}
$$

or, equivalently

$$
\dot{x}(t)=-A x(t)+B f(x(t-\tau))+c .
$$

Here, $x(t)=\left[x_{1}(t), x_{2}(t), \ldots, x_{n}(t)\right]^{T}$ is the neural state vector; $f(x(t-\tau))=\left[f_{1}\left(x_{1}(t-\tau)\right), f_{2}\left(x_{2}(t-\tau)\right), \ldots, f_{n}\left(x_{n}(t-\tau)\right)\right]^{T}$ denotes the activation function; $c=\left[c_{1}, c_{2}, \ldots, c_{n}\right]$ is the constant external input; and $A=\operatorname{diag}\left\{a_{1}, a_{2}, \ldots, a_{n}\right\}>0 \in \mathbb{R}^{n \times n}$ is a positive diagonal matrix. The scalar $\tau>0$ is a constant time delay. The interconnection matrix $B$ represents the delayed weight coefficients of the neurons. Furthermore, we assume that $f_{j}, j=1,2, \ldots, n$, satisfies the following assumption [22].

Assumption 1: The activation function $f(x)$ is continuous and bounded and it satisfies the following inequality:

$$
0 \leq \frac{f_{j}\left(s_{1}\right)-f_{j}\left(s_{2}\right)}{s_{1}-s_{2}} \leq l_{j}, \quad j=1,2, \ldots, n .
$$

for all $s_{1}, s_{2} \in \mathbb{R}, s_{1} \neq s_{2}$.

It should be pointed out that Assumption 1 guarantees there is an equilibrium point for HNN (2). This can be easily verified by employing the well-known Brouwer's fixed point theorem. Let $x^{*}=$ $\left[x_{1}^{*}, x_{2}^{*}, \ldots, x_{n}^{*}\right]$ be the equilibrium point. Then, in order to simplify the equation, we make the following transformation by the change of variables:

$$
y(t)=x(t)-x^{*} .
$$

Under this transformation, HNN (2) is rewritten as

$$
\dot{y}(t)=-A y(t)+B g(y(t-\tau))
$$

where

$$
g_{j}\left(y_{j}(t)\right)=f_{j}\left(y_{j}(t)+x_{j}^{*}\right)-f_{j}\left(x_{j}^{*}\right)
$$

By (4) and Assumption 1, it is not difficult to verify that

$$
g_{j}(0)=0,0 \leq \frac{g_{j}\left(y_{j}\right)}{y_{j}} \leq l_{j} \quad \forall y_{j} \neq 0, \quad j=1,2, \ldots, n .
$$

\section{MAIN RESUlT}

Manuscript received April 12, 2007; revised August 14, 2007; accepted October 12, 2007. This work was supported in part by the National Natural Science Foundation of China under Grant 60504008, the Program for New Century Excellent Talents in University, China, and the Research Grant Council under Grant HKU 7031/06P.

S. Mou, H. Gao, and W. Qiang are with the Department of Control Science and Engineering, Harbin Institute of Technology, Harbin, Heilongjiang Province 150001, China (e-mail: shaoshuaimou@gmail.com; hjgao@hit.edu.cn; qiangwy@hit.edu.cn).

J. Lam is with the Department of Mechanical Engineering, University of Hong Kong, Hong Kong 00852, China (e-mail: james.lam@hku.hk).

Digital Object Identifier 10.1109/TNN.2007.912593 resent our new delay-dependent asymptotic staIn this section, we present our new
ility criterion for delayed HNNs.

Theorem 1: Given an integer $m \geq 1$, the origin of the delayed $\mathrm{HNN}$ in (2) is the unique equilibrium point and it is globally asymptotically stable, if there exist positive-definite matrices $P \in \mathbb{R}^{n \times n}, Q \in$ $\mathbb{R}^{m n \times m n}, R \in \mathbb{R}^{n \times n}$, and $S=\operatorname{diag}\left\{s_{1}, s_{2}, \ldots, s_{n}\right\}$ such that

$$
\Theta=W_{P}^{T} \bar{P} W_{P}+W_{R}^{T} \bar{R} W_{R}+W_{Q}^{T} \bar{Q} W_{Q}+W_{S}^{T} \bar{S} W_{S}<0
$$


where

$$
\begin{aligned}
& \bar{P}=\left[\begin{array}{cc}
0 & P \\
P & 0
\end{array}\right] \quad \bar{R}=\left[\begin{array}{cc}
R & 0 \\
0 & -R
\end{array}\right] \\
& \bar{Q}=\left[\begin{array}{cc}
Q & 0 \\
0 & -Q
\end{array}\right] \quad \bar{S}=\left[\begin{array}{cc}
0 & S \\
S & -S
\end{array}\right] \\
& W_{R}=\left[\begin{array}{lll}
-\sqrt{\frac{\tau}{m}} A & 0_{n, m n} & \sqrt{\frac{\tau}{m}} B \\
\hline-\sqrt{\frac{m}{\tau}} I_{n} & \sqrt{\frac{m}{\tau}} I_{n} & 0_{n, m n}
\end{array}\right] \\
& W_{P}=\left[\begin{array}{ccc}
-A & 0_{n, m n} & B \\
I_{n} & 0_{n, m n} & 0_{n}
\end{array}\right] \\
& W_{S}=\left[\begin{array}{ccc}
0_{n, m n} & \frac{1}{\sqrt{2}} L & 0_{n} \\
0_{n, m n} & 0_{n} & \sqrt{2} I_{n}
\end{array}\right] \\
& W_{Q}=\left[\begin{array}{ccc}
I_{m n} & 0_{m n, n} & 0_{m n, n} \\
\hline 0_{m n, n} & I_{m n} & 0_{m n, n}
\end{array}\right] \\
& L=\operatorname{diag}\left\{l_{1}, l_{2}, \ldots, l_{n}\right\} .
\end{aligned}
$$

Proof: The uniqueness of the equilibrium point can be proved by the contradiction method similar in [22]. Now, we are in the position to show that the equilibrium point is globally asymptotically stable. At first, we choose a Lyapunov-Krasovskii functional candidate as

$$
V(t)=V_{1}(t)+V_{2}(t)+V_{3}(t)
$$

where

$$
\begin{aligned}
& V_{1}(t)=y(t)^{T} P y(t), \\
& V_{2}(t)=\int_{-\frac{\tau}{m}}^{0} \int_{t+v}^{t} \dot{y}^{T}(\omega) R \dot{y}(\omega) d \omega d v \\
& V_{3}(t)=\int_{t-\frac{\tau}{m}}^{t} \Upsilon(\omega)^{T} Q \Upsilon(\omega) d \omega
\end{aligned}
$$

and

$$
\Upsilon(\omega)=\left[\begin{array}{c}
y(\omega) \\
y\left(\omega-\frac{1}{m} \tau\right) \\
\vdots \\
y\left(\omega-\frac{m-1}{m} \tau\right)
\end{array}\right] .
$$

The derivatives of $V_{i}(t), i=1,2,3$, are given by

$$
\begin{aligned}
& \dot{V}_{1}(t)=2 y(t)^{T} P \dot{y}(t) \\
& \dot{V}_{2}(t)=\frac{\tau}{m} \dot{y}^{T}(t) R \dot{y}(t)-\int_{t-\frac{\tau}{m}}^{t} \dot{y}^{T}(\omega) R \dot{y}(\omega) d \omega \\
& \dot{V}_{3}(t)=\Upsilon(t)^{T} Q \Upsilon(t)-\Upsilon\left(t-\frac{\tau}{m}\right)^{T} Q \Upsilon\left(t-\frac{\tau}{m}\right) .
\end{aligned}
$$

From Jensen's inequality, we can easily get

$$
\begin{aligned}
-\int_{t-\frac{\tau}{m}}^{t} \dot{y}^{T}(\omega) R \dot{y}(\omega) d \omega & \leq-\frac{m}{\tau} \\
\times & {\left[y(t)-y\left(t-\frac{\tau}{m}\right)\right]^{T} R\left[y(t)-y\left(t-\frac{\tau}{m}\right)\right] . }
\end{aligned}
$$

From (5), for any scalar $s_{j} \geq 0$, it is clear that

$$
2 \sum_{j=1}^{n} s_{j} g_{j}\left(y_{j}(t-\tau)\right)\left[l_{j} y_{j}(t-\tau)-g_{j}\left(y_{j}(t-\tau)\right)\right] \geq 0
$$

or, equivalently

$$
2 g(y(t-\tau))^{T} S L y(t-\tau)-2 g(y(t-\tau))^{T} S g(y(t-\tau) \geq 0 .
$$

Using (3), (8), (9), and (10), we have

$$
\begin{aligned}
\dot{V}(t) \leq & 2 y(t)^{T} P[-A y(t)+B g(y(t-\tau))] \\
& +\frac{\tau}{m}[-A y(t)+B g(y(t-\tau))]^{T} R[-A y(t)+B g(y(t-\tau))] \\
& -\frac{m}{\tau}\left[y(t)-y\left(t-\frac{\tau}{m}\right)\right]^{T} R\left[y(t)-y\left(t-\frac{\tau}{m}\right)\right] \\
& +\Upsilon(t)^{T} Q \Upsilon(t)-\Upsilon\left(t-\frac{\tau}{m}\right)^{T} Q \Upsilon\left(t-\frac{\tau}{m}\right) \\
& +2 g(y(t-\tau))^{T} S L y(t-\tau)-2 g(y(t-\tau))^{T} S g(y(t-\tau) \\
= & \zeta(t)^{T} \Theta \zeta(t)
\end{aligned}
$$

where

$$
\zeta(t)=\left[\begin{array}{c}
\Upsilon(t) \\
y(t-\tau) \\
g(y(t-\tau))
\end{array}\right]
$$

and $\Theta$ is defined in (6).

On the other hand, condition (6) implies that there exists a positive e such that

$$
\Theta<\operatorname{diag}\{-\varepsilon I, 0, \ldots, 0,0\}
$$

We pre- and postmultiply this inequality by $\zeta^{T}(t)$ and $\zeta(t)$, respectively. Then, one can easily achieve

$$
\dot{V}(t) \leq \zeta(t)^{T} \Theta \zeta(t)<-\varepsilon\|y(t)\|^{2}
$$

which shows that the delayed HNN in (2) is asymptotically stable. This completes the proof.

Remark 1: Theorem 1 presents a new delay-dependent stability criterion for delayed HNNs by using a more general Lyapunov-Krasovskii functional in (7). Note that, even for $m=1$, the proposed result still demonstrates superiority over the main classical results in the literature. An illustrative example will be provided to show this in Section IV.

Remark 2: The reduced conservatism of Theorem 1 benefits from the construction of the new Lyapunov-Krasovskii functional in (7). The main idea is to fraction the delay, which constitutes the major difference from most existing results in the literature. Moreover, the conservatism reduction increases as the delay fractioning becomes thinner.

Remark 3: The delay fractioning idea can be extended to the timevarying delay case. We just need to modify our Lyapunov-Krasovskii functional by replacing $\tau$ with $\tau(t)(0 \leq \tau(t) \leq h, \dot{\tau} \leq \mu)$ to get LMI-based sufficient condition for HNN with time-varying delay.

If we choose the Lyapunov-Krasovskii functional as

$$
V(t)=y(t)^{T} P y(t)+\int_{t-\frac{\tau}{m}}^{t} \Upsilon(\omega)^{T} Q \Upsilon(\omega) d \omega
$$

then it is easy to obtain the following delay-independent stability condition.

Corollary 1: Given an integer $m \geq 1$, the origin of the delayed $\mathrm{HNN}$ in (2) is the unique equilibrium point and it is delay-independent globally asymptotically stable, if there exist positive-definite matrices $P \in \mathbb{R}^{n \times n}, Q \in \mathbb{R}^{m n \times m n}$, and $S=\operatorname{diag}\left\{s_{1}, s_{2}, \ldots, s_{n}\right\}$ such that

$$
\Omega=W_{P}^{T} \bar{P} W_{P}+W_{Q}^{T} \bar{Q} W_{Q}+W_{S}^{T} \bar{S} W_{S}<0
$$


where

$$
\begin{aligned}
\bar{P} & =\left[\begin{array}{cc}
0 & P \\
P & 0
\end{array}\right] \quad \bar{Q}=\left[\begin{array}{cc}
Q & 0 \\
0 & -Q
\end{array}\right] \\
\bar{S} & =\left[\begin{array}{cc}
0 & S \\
S & -S
\end{array}\right] \quad L=\operatorname{diag}\left\{l_{1}, l_{2}, \ldots, l_{n}\right\}, \\
W_{P} & =\left[\begin{array}{ccc}
-A & 0_{n, m n} & B \\
I_{n} & 0_{n, m n} & 0_{n}
\end{array}\right] \\
W_{S} & =\left[\begin{array}{lll}
0_{n, m n} & \frac{1}{\sqrt{2}} L & 0_{n} \\
0_{n, m n} & 0_{n} & \sqrt{2} I_{n}
\end{array}\right] \\
W_{Q} & =\left[\begin{array}{lll}
I_{m n} & 0_{m n, n} & 0_{m n, n} \\
\hline 0_{m n, n} & I_{m n} & 0_{m n, n}
\end{array}\right] .
\end{aligned}
$$

Proof: The derivative of (12) is

$$
\begin{aligned}
\dot{V}_{1}(t)= & 2 y(t)^{T} P \dot{y}(t)+\Upsilon(t)^{T} Q \Upsilon(t)-\Upsilon\left(t-\frac{\tau}{m}\right)^{T} Q \Upsilon\left(t-\frac{\tau}{m}\right) \\
\leq & 2 y(t)^{T} P[-A y(t)+B g(y(t-\tau))] \\
& +\Upsilon(t)^{T} Q \Upsilon(t)-\Upsilon\left(t-\frac{\tau}{m}\right)^{T} Q \Upsilon\left(t-\frac{\tau}{m}\right) \\
& +2 g(y(t-\tau))^{T} S L y(t-\tau)-2 g(y(t-\tau))^{T} S g(y(t-\tau)) \\
\leq & \zeta(t)^{T} \Omega \zeta(t)
\end{aligned}
$$

where $\zeta(t)$ is as defined in (11) and $\Omega$ in (13). Then, using similar method in Theorem 1, we complete the proof.

Remark 4: Though not in ordinary form of LMI, Theorem 1 and Collary 1 are indeed in the standard LMI form, which can be easily solved by the standard software. Moreover, this form simplified as $W_{1}^{T} X W_{1}+W_{2}^{T} Y W_{2}<0$ is more laconic. It expresses the LMI in several parts, each of which has a symmetric structure with the matrix variable to be determined in center.

\section{ILLUSTRATIVE EXAMPLES}

In this section, an example is provided to illustrate the advantage of Theorem 1 by comparing it with recently reported results on delaydependent asymptotic stability of delayed HNNs.

Example 1: Consider the following third-order delayed HNN:

$$
\begin{aligned}
A & =\left[\begin{array}{ccc}
4.1989 & 0 & 0 \\
0 & 0.7160 & 0 \\
0 & 0 & 1.9985
\end{array}\right] \\
B & =\left[\begin{array}{ccc}
-0.1052 & -0.5069 & -0.1121 \\
-0.0257 & -0.2808 & 0.0212 \\
0.1205 & -0.2153 & 0.1315
\end{array}\right]
\end{aligned}
$$

with

$$
L=\operatorname{diag}\{0.4129,3.8993,1.0160\} \text {. }
$$

Our purpose is to find the maximum allowable delay $\tau_{\max }$ such that the delayed HNN in (2) is globally asymptotically stable. Computational results are shown in Table I, which summarizes the obtained maximum allowable delays by using the previously published methods and our new result for various fractionings.

Table I shows that for no fractioning $(m=1)$, the new criterion given in Theorem 1 is still less conservative than the previous results, which corresponds to Remark 1 . Moreover, for $m>1$, the conservatism reduction proves to be more obvious. However, it should be noted that although conservatism is reduced as the fractioning becomes thinner, there is no significant improvement after $m=5$.
TABLE I

MaXimum AlLOWABLe Delays COMPARISON

\begin{tabular}{c|c}
\hline Method & $\tau_{\max }$ \\
\hline Ye and Michel, 1994, [23] & 0.4121 \\
\hline Xu and Lam, 2005, [21] & 1.7484 \\
\hline Xu and Lam, 2006, [22] & 1.7644 \\
\hline Theorem 1, $m=1$ & 2.14 \\
\hline Theorem 1, $m=2$ & 2.48 \\
\hline Theorem 1, $m=3$ & 2.54 \\
\hline Theorem 1, $m=4$ & 2.57 \\
\hline Theorem 1, $m=5$ & 2.581 \\
\hline Theorem 1, $m=10$ & 2.596 \\
\hline Theorem $1, m=15$ & 2.597
\end{tabular}

\section{CONCLUSION}

By defining a new Lyapunov-Krasovskii functional, an improved delay-dependent asymptotic stability criterion has been obtained for a class of delayed HNNs. The proposed condition is given in terms of LMIs and thus can be readily solved via standard numerical software. The merit of the proposed condition lies in its reduced conservatism, which is based on a time delay fractioning approach. The result proves to become less conservative as the fractioning goes thinner. Finally, a numerical example has been provided to demonstrate the effectiveness of the proposed criterion. The method is expected to be further extended to other neural networks or complex networks [4], [5], [7], [10], [11], [15], [18], which is under our investigation.

\section{REFERENCES}

[1] E.-K. Boukas and N. F. Al-Muthairi, "Delay-dependent stabilization of singular linear systems with delays," Int. J. Innov. Comput. Inf. Control, vol. 2, no. 2, pp. 283-291, 2006.

[2] J. Cao, "An estimation of the domain of attraction and convergence rate for Hopfield continuous feedback neural networks," Physica A, vol. 325, no. 5-6, pp. 370-374, 2004.

[3] J. Cao and J. Wang, "Global asymptotic stability of a general class of recurrent neural networks with time-varying delays," IEEE Trans. Circuits Syst. I, Fundam. Theory Appl., vol. 50, no. 1, pp. 34-44, Jan. 2003.

[4] W. Chen and X. Lu, "New delay-dependent exponential stability criteria for neural networks with variable delays," Physica A, vol. 351, no. $1-2$, pp. 53-58, 2006.

[5] W. Chen and W. Zheng, "Global asymptotic stability of a class of neural networks with distributed delays," IEEE Trans. Circuits Syst. I, Reg. Papers, vol. 53, no. 3, pp. 644-652, Mar. 2006.

[6] J. Farrell and A. Michel, "A synthesis procedure for Hopfield's continuous-time associative memory," IEEE Trans. Circuits Syst., vol. 37, no. 7, pp. 877-884, Jul. 1990.

[7] H. Gao, J. Lam, and G. Chen, "New criteria for synchronization stability of general complex dynamical networks with coupling delays," Phys. Lett. A, vol. 360, no. 2, pp. 263-273, 2006.

[8] H. Gao and C. Wang, "Comments and further results on 'A descriptor system approach to $H_{\infty}$ control of linear time-delay systems'," IEEE Trans. Autom. Control, vol. 48, no. 3, pp. 520-525, Mar. 2003.

[9] H. Gao and C. Wang, "A delay-dependent approach to robust $H_{\infty}$ filtering for uncertain discrete-time state-delayed systems," IEEE Trans. Signal Process., vol. 52, no. 6, pp. 1631-1640, Jun. 2004.

[10] Y. He, Q. Wang, and W. Zheng, "Global robust stability for delayed neural networks with polytopic type uncertainties," Chaos Solitons Fractals, vol. 26, pp. 1349-1354, 2005.

[11] Y. He, M. Wu, and J. H. She, "An improved global asymptotic stability criterion for delayed cellular neural networks," IEEE Trans. Neural Netw., vol. 17, no. 1, pp. 250-252, Jan. 2006.

[12] H. Huang, D. W. C. Ho, and J. Lam, "Stochastic stability analysis of fuzzy Hopfield neural networks with time-varying delays," IEEE Trans. Circuits Syst. II, Exp. Briefs, vol. 52, no. 5, pp. 251-255, May 2005 
[13] X. Liu, K. L. Teo, and B. Xu, "Exponential stability of impulsive highorder Hopfield-type neural networks with time-varying delays," IEEE Trans. Neural Netw., vol. 16, no. 6, pp. 1329-1339, Jun. 2005.

[14] X. Y. Lou and B. Cui, "New LMI conditions for delay-dependent asymptotic stability of delayed Hopfield neural networks," Neurocomputing, vol. 69, no. 16-18, pp. 2374-2378, 2006.

[15] V. Singh, "A generalized LMI-based approach to the global asymptotic stability of delayed cellular neural networks," IEEE Trans. Neural Netw., vol. 15, no. 1, pp. 223-225, Jan. 2004.

[16] Z. Wang, Y. Liu, K. Fraser, and X. Liu, "Stochastic stability of uncertain Hopfield neural networks with discrete and distributed delays," Phys. Lett. A, vol. 354, no. 4, pp. 288-297, 2006.

[17] Z. Wang, Y. Liu, and X. Liu, "On global asymptotic stability of neural networks with discrete and distributed delays," Phys. Lett. A, vol. 345, no. 4-6, pp. 299-308, 2005.

[18] Z. Wang, Y. Liu, and X. Liu, "Stability analysis for stochastic Cohen-Grossberg neural networks with mixed time delays," IEEE Trans. Neural Netw., vol. 17, no. 3, pp. 814-820, May 2006.

[19] Z. Wang, H. Shu, J. Fang, and X. Liu, "Robust stability for stochastic Hopfield neural networks with time delays," Nonlinear Anal.: Real World Appl., vol. 7, no. 5, pp. 1119-1128, 2006.

[20] B. Xu, X. Liu, and X. Liao, "Global exponential stability of high order Hopfield type neural networks," Appl. Math. Comput., vol. 174, no. 1, pp. 98-116, 2006.

[21] S. Xu, J. Lam, D. W. C. Ho, and Y. Zou, "Novel global asymptotic stability criteria for delayed cellular neural networks," IEEE Trans. Circuits Syst. II, Exp. Briefs, vol. 52, no. 6, pp. 349-353, Jun. 2005.

[22] S. Xu, J. Lam, and D. W. C. Ho, "A new LMI condition for delaydependent asymptotic stability of delayed Hopfield neural networks," IEEE Trans. Circuits Syst. II. Exp. Briefs, vol. 53, no. 3, pp. 230-234, Mar. 2006.

[23] H. Ye, A. Michel, and K. Wang, "Global stability and local stability of Hopfield neural networks with delays," Phys. Rev. E, Stat. Phys. Plasmas Fluids Relat. Interdiscip. Top., vol. 50, pp. 4026-4213, 1994.

[24] F. Zhang and H. Huo, "Global stability of delayed Hopfield neural networks under dynamical thresholds," Discrete Dyn. Nature Soc., vol. 1, pp. 1-17, 2005, 2005.

[25] H. Zhao, "Global asymptotic stability of Hopfield neural network involving distributed delays," Neural Netw., vol. 17, no. 1, pp. 47-53, 2004.

\section{Energy Function and Energy Evolution on Neuronal Populations}

\author{
Rubin Wang, Zhikang Zhang, and Guanrong Chen
}

\begin{abstract}
Based on the principle of energy coding, an energy function of a variety of electric potentials of a neural population in cerebral cortex is formulated. The energy function is used to describe the energy evolution of the neuronal population with time and the coupled relationship between neurons at the subthreshold and the suprathreshold states. The Hamiltonian motion equation with the membrane potential is obtained from the neuroelectrophysiological data contaminated by Gaussian white noise. The results of this research show that the mean membrane potential is the exact solution of the motion equation of the membrane potential developed in a previously published paper. It also shows that the Hamiltonian energy function derived in this brief is not only correct but also effective. Particularly, based on the principle of energy coding, an interesting finding is that in some subsets of neurons, firing action potentials at the suprathreshold and some others simultaneously perform activities at the subthreshold level in neural ensembles. Notably, this kind of coupling has not been found in other models of biological neural networks.
\end{abstract}

Index Terms-Coupled neural population, energy coding, energy evolution, Hamiltonian function.

\section{INTRODUCTION}

Due to the limitations in current biophysical models of neural coding, research into the mechanisms of neural information processing remains a challenge [1]-[3], thereby the fundamental principles of neural information processing underlying cognitive processes in the brain are still not completely understood today.

Regarding neural information processing, the basic theory of energy coding has received strong supports from many neuroelectrophysiological experiments [4]-[11], [13]-[16], and a significant expansion on energy coding appear in [7]. Two concerned issues pertaining to the aforementioned research results are considered in this brief.

1) Neuronal activities at the subthreshold and the suprathreshold states are separately described and discussed in their mathematical models. The fact is that most activities of neurons at both of these threshold states are mutually coupled, so this separate description does not agree with the real neuronal activities. For this reason, the coupled relationship between neurons at the subthreshold and suprathreshold states is taken into account in this brief, and under such coupling configuration some quantitative expressions of energy coding are obtained.

2) The Hamiltonian energy function describing the collective activities of electric potentials of a whole neural population is derived and analyzed, improving the previously published results devoted only to a single neuron [5], [7]. Therefore, the results reported in this brief are quite universal and significant.

Manuscript received April 14, 2007; revised June 5, 2007; accepted October 12, 2007. This work was supported by the National Natural Science Foundation of China under Grant 10672057.

R. Wang and Z. Zhang are with the Institute for Brain Information Processing and Cognitive Neurodynamics, School of Information Science and Engineering, East China University of Science and Technology, Shanghai 200237, P. R. China (e-mail: rbwang@163.com).

G. Chen is with the Department of Electronic Engineering, City University of Hong Kong, Kowloon, Hong Kong SAR, P. R. China.

Digital Object Identifier 10.1109/TNN.2007.914177 